\title{
NOTES ON ORTHOGONAL POLYNOMIALS IN TWO VARIABLES
}

\author{
HEINRICH LARCHER
}

In a paper which will be referred to henceforth as [1] Groebner has observed that a necessary and sufficient condition for a system of functions $\phi_{1}(x), \phi_{2}(x), \cdots, \phi_{n}(x), \cdots$ to be orthogonal in an interval $[a, b]$ is that for $n=1,2, \ldots$ the integral

$$
I_{n}=\int_{a}^{b}\left[c_{1} \phi_{1}(x)+c_{2} \phi_{2}(x)+\cdots+c_{n-1} \phi_{n-1}(x)+\phi_{n}(x)\right]^{2} d x
$$

achieves its minimum value for $c_{1}=c_{2}=\cdots=c_{n-1}=0$. Making use of this minimum condition he has shown in the case of the Legendre Polynomials how the calculus of variations can be used to obtain these polynomials. $\S 4,5$ and 6 of [1] suggest that the author was particularly interested in extending this method to obtaining systems of orthogonal polynomials in two variables for two dimensional regions. For a triangular and a circular region the work was performed. The results are hardly satisfactory as in these cases the calculus of variations yielded systems of polynomials which had to be subjected to an additional process of orthogonalization to obtain the desired systems. In this paper it will be shown that for certain regions in the $x-y$ plane the calculus of variations can be employed to obtain the polynomials of the respective orthogonal systems immediately.

Before starting out we state the problem explicitly. The object is to orthogonalize the system

$$
1, y, x, y^{2}, x y, x^{2}, y^{3}, x y^{2}, \cdots
$$

with respect to some region $R$. For doing this several processes of orthogonalization are available. Among them the method of the calculus of variations, if it can be employed, has the advantage of yielding expressions in closed form for the polynomials.

From now on $p_{m n}(x, y)$ stands for a polynomial in $x$ and $y$ of total degree $m+n$ which contains the term $x^{m} y^{n}$ with a nonvanishing coefficient, and may contain terms $x^{s} y^{t}$ with $s+t=m+n$ and $s<m$, as well as any terms of lower degrees - or by making use of (2) any terms with products of powers of $x$ and $y$ which appear on the left of $x^{m} y^{n}$.

In order to save writing we introduce an order relation in the set of

Received by the editors January 23, 1958 and, in revised form, September 30, 1958. 
pairs of numbers $(m, n)$ with $m, n=0,1,2, \cdots$ which correspond to the subscripts of the $p_{m n}(x, y)$. The order relation we denote by $<$ and is defined to mean:

$$
(s, t)<(m, n)=\left\{\begin{array}{l}
s+t<m+n \text { or } \\
s+t=m+n \text { and } s<m, \text { with equality }
\end{array}\right.
$$

$(s, t)=(m, n)$ holding if and only if $s=m$ and $t=n$.

The problem is to find a system of polynomials $\left\{p_{m n}(x, y)\right\}$ with $m, n=0,1,2, \cdots$ which are orthogonal with respect to some region $R$, namely

$$
\int_{R} p_{m n}(x, y) p_{s t}(x, y) d x d y= \begin{cases}0 & \text { for }(m, n) \neq(s, t), \\ \pi_{m n} \text { for }(m, n)=(s, t) .\end{cases}
$$

Now it is easy to show that if for a region $R$ an orthogonal system of polynomials $\left\{p_{m n}(x, y)\right\}$ as defined exists it is unique save for a multiplicative constant for the polynomials.

Proof. Let $\left\{q_{m n}(x, y)\right\}$ be another system of polynomials that satisfy the orthogonality relations $(3)$, where $q_{m n}(x, y)$ is defined like $p_{m n}(x, y)$ and, moreover, that the coefficient of the leading term, i.e. the term containing $x^{m} y^{n}$, is the same for both. We form

$$
\int_{R}\left(q_{m n}-p_{m n}\right)^{2} d x d y
$$

where we wrote briefly $p_{m n}$ and $q_{m n}$ instead of $p_{m n}(x, y)$ and $q_{m n}(x, y)$, and show that it vanishes for arbitrary $m$ and $n$. If we replace after multiplying out the integrand of (4) in $2 q_{m n} p_{m n}$ first $p_{m n}$ by a linear combination of the $q_{i j}$ with $(0,0) \leqq(i, j) \leqq(m, n)$ and then $q_{m n}$ by a corresponding expression of the $p_{i j}$ we find that

$$
\int_{R}\left(p_{m n}^{2}-q_{m n}^{2}\right) d x d y=\int_{R}\left(q_{m n}^{2}-p_{m n}^{2}\right) d x d y,
$$

from which follows the vanishing of (4). Consequently $q_{m n} \equiv p_{m n}$ for $m, n=0,1,2, \cdots$.

For the following we assume the region $R$ to be bounded or partially bounded by the curves $x=\phi_{1}(y)$ and $x=\phi_{2}(y)$ which we assume to be single-valued and continuous functions in an interval $[a, b]$ and that

$$
\phi_{1}(y) \leqq \phi_{2}(y) \text { for } a \leqq y \leqq b .
$$

Additional conditions on $\phi_{1}(y)$ and $\phi_{2}(y)$ will be imposed as we go along. 
Lemma. If for a given region $R, p_{m}(x, y)$ is the polynomial in $x$ and $y$ of total degree $m$ and degree $m$ in $x$ of the orthogonal system in

$$
\left[\phi_{1}(y), \phi_{2}(y)\right]
$$

and $p_{n}(y)$ is the polynomial in $y$ of degree $n$ of the orthogonal system in $[a, b]$ with the weight function $w_{m}(y)=\int_{\phi_{1}(y)}^{\phi_{2}(y)}\left[p_{m}(x, y)\right]^{2} d x$, then $p_{m}(x, y) p_{n}(y)$ is a polynomial $p_{m n}(x, y)$ as defined previously and the polynomials of the system $\left\{p_{m}(x, y) p_{n}(y)\right\}$ with $m, n=0,1,2, \ldots$ satisfy the orthogonality relations (3).

Proof. The first part of the conclusion is obvious and hence we write

$$
p_{m}(x, y) p_{n}(y)=p_{m n}(x, y)
$$

By hypothesis

$p_{n}(y) p_{t}(y) \int_{\phi_{1}(y)}^{\phi_{2}(y)} p_{m}(x, y) p_{s}(x, y) d x=0$ for $s \neq m$ and arbitrary $n$ and $t$.

In case of $s=m$ and $n \neq t$

$$
\int_{a}^{b} w_{m}(y) p_{n}(y) p_{t}(y) d y=0 .
$$

The two integrals are equivalent to (3).

From the lemma it is evident that $p_{m}(x, y)$ is a polynomial of at most degree $m$ in $y$ and that in certain cases it might even be a function of $x$ alone. Furthermore we note that the requirement for $p_{m}(x, y)$ and $p_{n}(y)$ to be polynomials lays down restrictions on $\phi_{1}(y)$ and $\phi_{2}(y)$. We shall now proceed to obtain the orthogonal systems of polynomials $\left\{p_{m}(x, y)\right\}$ and $\left\{p_{n}(y)\right\}$, note the restrictions that have to be imposed on $\phi_{1}(y)$ and $\phi_{2}(y)$ explicitly, and hence infer for which regions $R$ this method yields a system of orthogonal polynomials $\left\{p_{m n}(x, y)\right\}$.

It is well known that the polynomial $p_{m}(x, y)$ of the orthogonal system in $\left[\phi_{1}(y), \phi_{2}(y)\right]$ is the "Legendre Polynomial"

$$
p_{m}(x, y)=\frac{m !}{(2 m) !} \frac{\partial^{m}}{\partial x^{m}}\left[\left(x-\phi_{1}(y)\right)^{m}\left(x-\phi_{2}(y)\right)^{m}\right],
$$

where for the time being the constant factor is chosen so that the coefficient of the leading term is unity.

(7) will be a polynomial of at most degree $m$ in $y$ by requiring that $\phi_{1}(y)$ and $\phi_{2}(y)$ be either

(i) constant or linear, or

(ii) both of the form $c y+d \pm\left(e y^{2}+f y+g\right)^{1 / 2}$, 
with the plus and minus sign for $\phi_{2}(y)$ and $\phi_{1}(y)$, respectively, in agreement with (5). It is hardly necessary to remark that the restrictions on $e, f$ and $g$ are such that $e y^{2}+f y+g \geqq 0$ for $a \leqq y \leqq b$ and that (ii) does not reduce to (i).

Proof. As (ii) actually comprises (i) it suffices to verify it for the former case.

$$
\left[x-\phi_{1}(y)\right]^{m}\left[x-\phi_{2}(y)\right]^{m}=\left[(x-c y-d)^{2}-\left(e y^{2}+f y+g\right)\right]^{m}
$$

is a polynomial in $x$ and $y$ of degree $2 m$ that contains the term $x^{2 n}$. Differentiating it $m$-times with respect to $x$ yields a polynomial of degree $m$. It is understood that henceforth $\phi_{1}(y)$ and $\phi_{2}(y)$ are subject to the restrictions $(8)$.

The norm of $p_{m}$ - for $p_{m}(x, y)$ and $p_{n}(y)$ we shall frequently write briefly $p_{m}$ and $p_{n}$ - which is obtained by integrating by parts and observing that at $x=\phi_{1}(y)$ and $x=\phi_{2}(y)$ all terms but the last vanish is

$$
\int_{\phi_{1}(y)}^{\phi_{2}(y)} p_{m}^{2} d x=\frac{[m !]^{4}(2 m+1)}{[(2 m+1) !]^{2}}\left[\phi_{2}(y)-\phi_{1}(y)\right]^{2 m+1} .
$$

Because of (5), $\left[\phi_{2}(y)-\phi_{1}(y)\right]^{2 m+1} \geqq 0$ and is except for an irrelevant numerical factor the weight function $w_{m}(y)$.

According to $[1$, pp. 42 and 43$]$ the polynomial $p_{n}(y)$ of the orthogonal system in $[a, b]$ with the weight function $w_{m}(y)$, properly restricted if necessary, is the solution of the minimum problem

$$
\begin{aligned}
\int_{a}^{b}\left[\phi_{2}(y)-\phi_{1}(y)\right]^{2 m+1}\left[p_{n}(y)\right]^{2} d y & =\min , \\
\frac{d^{n} p_{n}}{d y^{n}} & =n !
\end{aligned}
$$

where for the time being the coefficient of $y^{n}$ is chosen to be unity.

As can be seen from $(10) p_{n}(y, m)$ would be a more appropriate notation than $p_{n}(y)$. We shall still use the latter as it is understood that finally $p_{n}(y)$ has to be multiplied by $p_{m}(x, y)$ to form $p_{m n}(x, y)$.

To solve (10) with the additional restriction (11) we make use of the "Lagrange multiplier" method. Thus we multiply (11) by an arbitrary function $\lambda_{n}(y)$ and form the integral

$$
I_{n}=\int_{a}^{b}\left(\phi_{2}-\phi_{1}\right)^{2 m+1}\left[p_{n}^{2}+\lambda_{n}\left(p_{n}^{(n)}-n !\right)\right] d y,
$$

where $p_{n}^{(n)}$ denotes the $n$th derivative of $p_{n}(y)$, while the remaining abbreviations are evident.

The first variation of $I_{n}$, i.e. 


$$
\delta I_{n}=\int_{a}^{b}\left[2 p_{n}\left(\phi_{2}-\phi_{1}\right)^{2 m+1} \delta p_{n}+\lambda_{n}\left(\phi_{2}-\phi_{1}\right)^{2 m+1} \delta p_{n}^{(n)}\right] d y
$$

must vanish for arbitrary variations $\delta p_{n}$.

Putting $\lambda_{n}\left(\phi_{2}-\phi_{1}\right)^{2 m+1}=\Lambda_{n}$ and integrating by parts to change $\delta p_{n}^{(n)}$ to $\delta p_{n}$, we obtain

$$
\begin{aligned}
\delta I_{n}= & {\left[\Lambda_{n} \delta p_{n}^{(n-1)}-\Lambda_{n}^{\prime} \delta p_{n}^{(n-2)}+\cdots+(-1)^{n-1} \Lambda_{n}^{(n-1)} \delta p_{n}\right]_{a}^{b} } \\
& +\int_{a}^{b}\left[2 p_{n}\left(\phi_{2}-\phi_{1}\right)^{2 m+1}+(-1)^{n} \Lambda_{n}^{(n)}\right] \delta p_{n} d y=0 .
\end{aligned}
$$

The required vanishing of all terms yields Euler's differential equation

$$
p_{n}=\frac{(-1)^{n-1}}{2} \frac{1}{\left(\phi_{2}-\phi_{1}\right)^{2 m+1}} \frac{d^{n}}{d y^{n}}\left[\lambda_{n}\left(\phi_{2}-\phi_{1}\right)^{2 m+1}\right],
$$

which together with (11) gives the differential equation of order $2 n$ for $\lambda_{n}$

$$
\frac{d^{n}}{d y^{n}}\left\{\frac{1}{\left(\phi_{2}-\phi_{1}\right)^{2 m+1}} \frac{d^{n}}{d y^{n}}\left[\lambda_{n}\left(\phi_{2}-\phi_{1}\right)^{2 m+1}\right]\right\}=(-1)^{n} 2 n !
$$

and the $2 n$ boundary conditions

$$
\frac{d^{i}}{d y^{i}}\left[\lambda_{n}(y)\left(\phi_{2}-\phi_{1}\right)^{2 m+1}\right]=0 \text { for } y=a \text { and } y=b,
$$

$i=0,1, \cdots, n-1$ and any $m$.

Our problem is solved if we can find $\lambda_{n}(y)$ for a particular region $R$ so that it satisfies (13) and (14). Recalling the restrictions (5) and (8) on $\phi_{1}(y)$ and $\phi_{2}(y)$

$$
\lambda_{n}=k_{n}^{\prime}(y-a)^{n}(y-b)^{n},
$$

where $k_{n}^{\prime}$ is a constant whose value is of no importance at this stage, is as is easily verified the solution for the following regions:

(i) $\phi_{2}(y)-\phi_{1}(y)=k$-in all four cases $k$ denotes a positive constant-, which corresponds to a parallelogram with two sides parallel to the $x$-axis;

(16b) (ii) $\phi_{2}(y)-\phi_{1}(y)=k(y-a)$ or $k(b-y)$, which corresponds to a triangle with one side parallel to the $x$-axis; 
(16c) (iii) $\phi_{2}(y)-\phi_{1}(y)=2 k\left[-y^{2}+(a+b) y-a b\right]^{1 / 2}$, which according to (8) case (ii) for a particular $c$ and $d$ corresponds to the ellipse $(x-c y-d)^{2}=k^{2}\left[-y^{2}+(a+b) y-a b\right]$;

(16d) (iv) $\phi_{2}(y)-\phi_{1}(y)=2 k(y-a)^{1 / 2}$, which for a particular $c$ and $d$ corresponds to the parabolic region bounded by $(x-c y-d)^{2}$ $=k^{2}(y-a)$ and $y=b$. The same holds if $(y-a)^{1 / 2}$ and $y=b$ are replaced by $(b-y)^{1 / 2}$ and $y=a$ respectively.

It is to be noted that (16c) covers any ellipse in the $x-y$ plane and that (16d) holds for any parabola whose axis is not parallel to the $x$-axis. This last case can be dealt with by interchanging $x$ and $y$ in the derivation.

The expressions (15) and one of (16) introduced in (12) yield the desired polynomials $p_{n}(y)$ for the appropriate region. Finally, the constants accompanying the polynomials $p_{m}(x, y)$ and $p_{n}(y)$ are denoted by $k_{m}$ and $k_{n}$, respectively, and may be chosen suitably for particular regions.

Before considering two examples we summarize the results. Subject to the restrictions (8) and (16a, b, c, d) on $\phi_{1}(y)$ and $\phi_{2}(y)$ systems of orthogonal polynomials for "admissible" regions $R$ are given by

$$
p_{m n}(x, y)=p_{m}(x, y) p_{n}(y), \quad m, n=0,1,2, \cdots,
$$

where

$$
p_{m}(x, y)=k_{m} \frac{\partial^{m}}{\partial x^{m}}\left[\left(x-\phi_{1}(y)\right)^{m}\left(x-\phi_{2}(y)\right)^{m}\right]
$$

and

$p_{n}(y)=k_{n} \frac{1}{\left[\phi_{2}(y)-\phi_{1}(y)\right]^{2 m+1}} \frac{d^{n}}{d y^{n}}\left[(y-a)^{n}(y-b)^{n}\left(\phi_{2}(y)-\phi_{1}(y)\right)^{2 m+1}\right]$.

Examples. (i) For the triangle with vertices $(0,0),(1,0)$ and $(0,1)$ the polynomials are

$p_{m n}=\frac{1}{2^{m+n} m ! n !} \frac{1}{(1-y)^{2 m+1}} \frac{d^{n}}{d y^{n}}\left[y^{n}(1-y)^{n+2 m+1}\right] \frac{\partial^{m}}{\partial x^{m}}\left[x^{m}(x+y-1)^{m}\right]$.

(ii) For the circle $x^{2}+y^{2}=1$

$$
p_{m n}=\frac{1}{2^{m+n} m ! n !} \frac{1}{\left(y^{2}-1\right)^{m+1 / 2}} \frac{d^{n}}{d y^{n}}\left(y^{2}-1\right)^{m+n+1 / 2} \frac{\partial^{m}}{\partial x^{m}}\left(x^{2}+y^{2}-1\right)^{m} .
$$

The polynomials for the circle can be found in [2], while the closed 
form expressions for the polynomials for the triangle, for example, are believed not to have been known previously.

Certain properties of the polynomials of an orthogonal system for which expressions in closed form exist can be derived easily. This, in fact, motivates the search for them. For instance, for all the systems obtained above the generating function can be obtained by means of Lagrange's formula. For the unit circle the work is outlined in [2].

\title{
REFERENCES
}

1. W. Groebner, Ueber die Konstruktion von Systemen orthogonaler Polynome in ein- und zwei-dimensionalen Bereichen, Monatsh. Math. vol. 52 (1948) pp. 38-54.

2. F. Didon, Développements sur certaines séries de polynomes a un nombre quelconque de variables, Ann. Sci. Ecole Norm. Sup. vol. 7 (1870) pp. 247-268.

Michigan State University

\section{A COUNTER-EXAMPLE TO A STATEMENT ON CON- FORMAL MAPPING OF RIEMANN SURFACES}

\author{
JAMES A. JENKINS ${ }^{1}$
}

A few years ago H. L. Royden [3] asserted a result which in the nomenclature of the author [2] is stated as follows.

Let $\Re$ be a finite oriented Riemann surface, $Q(z) d z^{2}$ a positive quadratic differential on $\Re, \Delta$ an admissible domain with respect to $Q(z) d z^{2}$. Then the identity is the only conformal mapping $f$ of $\Delta$ into $\Re$ which has all the following properties:

(i) there is an interior point of $\Delta$ which is a fixed point of $f$;

(ii) the poles of $Q(z) d z^{2}$ which lie in $\Delta$ are fixed points of $f$ and near a pole of order $k \geqq 2$ the mapping has the form

$$
f(z)=z+a z^{k}+\cdots
$$

in terms of a local uniformizing parameter $z$ at the pole;

(iii) $f$ admits an admissible homotopy into the identity.

We will give now a simple example to show this assertion incorrect.

Let us denote by $\alpha$ the quantity $\left(e^{2 \pi} /\left(e^{2 \pi}-1\right)\right)^{1 / 2}$ and by $D$ the domain on the $z$-sphere exterior to the circle $|z-\alpha|=1$ (i.e., containing the point at infinity). Let $f_{\theta}(z)$ be the function defined in $[2$,

Received by the editors October 17, 1958.

${ }^{1}$ Research supported in part by the National Science Foundation, Mathematics Section, through the University of Notre Dame and by the Office of Ordnance Research under contract No. DA-36-034-ORD-2453 through the Institute for Advanced Study. 